\title{
Modeling Imprecise and Bipolar Algebraic and Topological Relations using Morphological Dilations
}

https://doi.org/10.1515/mathm-2020-0107

Received August 14, 2020; accepted March 7, 2021

\begin{abstract}
In many domains of information processing, such as knowledge representation, preference modeling, argumentation, multi-criteria decision analysis, spatial reasoning, both vagueness, or imprecision, and bipolarity, encompassing positive and negative parts of information, are core features of the information to be modeled and processed. This led to the development of the concept of bipolar fuzzy sets, and of associated models and tools, such as fusion and aggregation, similarity and distances, mathematical morphology. Here we propose to extend these tools by defining algebraic and topological relations between bipolar fuzzy sets, including intersection, inclusion, adjacency and RCC relations widely used in mereotopology, based on bipolar connectives (in a logical sense) and on mathematical morphology operators. These definitions are shown to have the desired properties and to be consistent with existing definitions on sets and fuzzy sets, while providing an additional bipolar feature. The proposed relations can be used for instance for preference modeling or spatial reasoning. They apply more generally to any type of functions taking values in a poset or a complete lattice, such as L-fuzzy sets.
\end{abstract}

Keywords: Bipolar fuzzy sets, topological relations, adjacency, mereotopology, region connection calculus, mathematical morphology operators.

MSC: 03B52, 03E72, 06B23, 68T

\section{Introduction}

In many domains, such as knowledge representation, preference modeling, argumentation, multi-criteria decision analysis, spatial reasoning, image understanding, both vagueness or imprecision and bipolarity, encompassing positive and negative parts of information, are core features of the information to be modeled and processed. Bipolarity corresponds to a recent trend in contemporary information processing, both from a knowledge representation point of view, and from a processing and reasoning one. It allows distinguishing between (i) positive information, which represents what is guaranteed to be possible, for instance because it has already been observed or experienced, and (ii) negative information, which represents what is impossible or forbidden, or surely false [22]. This domain has recently motivated work in several directions, for instance for applications in knowledge representation, preference modeling, argumentation, multi-criteria decision analysis, cooperative games, among others [22]. Three types of bipolarity are distinguished in [24]: (i) symmetric univariate, where a unique totally ordered scale covers the range from negative (not satisfactory) to positive (satisfactory) information (e.g. modeled by probabilities); (ii) symmetric bivariate, where two separate scales are linked together and concern related information (e.g. modeled by belief functions); (iii) asymmetric or heterogeneous, where two types of information are not necessarily linked together and may

^Corresponding Author: Isabelle Bloch: Sorbonne Université, CNRS, LIP6, Paris, France, on leave from LTCI, Télécom Paris, Institut Polytechnique de Paris, France, E-mail: isabelle.bloch@sorbonne-universite.fr, isabelle.bloch@telecom-paris.fr 
come from different sources. This last type is particularly interesting in image interpretation and spatial reasoning. In order to include the imprecise nature of information, fuzzy and possibilistic formalisms for bipolar information have been proposed (see e.g. [24]). This led to the development of the concept of bipolar fuzzy sets, and of associated models and tools, such as fusion and aggregation, similarity and distances, mathematical morphology, etc.

Here we propose to extend this set of tools by defining algebraic and topological relations between bipolar fuzzy sets, including intersection, inclusion, adjacency and relations of region connection calculus (RCC) widely used in mereotopology. Let us recall that mereology is concerned with part-whole relations, while mereotopology adds topology and studies topological relations where regions (not points) are the primitive objects, useful for qualitative spatial reasoning, see e.g. [1] and the references therein. Formal definitions are proposed, based on bipolar connectives and on mathematical morphology operators (here we consider only the deterministic part of mathematical morphology and use mostly dilations). Links with previous works on sets with broad boundaries [14] or egg-yolk [15, 27, 33] models are established. The proposed definitions are shown to have the desired properties and to be consistent with existing definitions on sets and fuzzy sets, while providing an additional bipolar feature. The usefulness of morphological operators in this construction can be found in the algebraic and unifying setting they offer, and in the properties they induce. The proposed relations can be used for instance for preference modeling or spatial reasoning, accounting for both bipolarity and imprecision. Any type of bipolar fuzzy set is considered, and the proposed definitions are not restricted to objects with indeterminate or broad boundaries as in the egg-yolk [15] or 9-intersection [14] models. This paper extends a preliminary version in [11] with more details, proofs, examples, and comparisons.

In Section 2, definitions of bipolar fuzzy sets and bipolar connectives are summarized. Extensions of basic set theoretical (algebraic) relations to bipolar fuzzy sets are given in Section 3. Mathematical morphology operators are recalled in Section 4. Adjacency is addressed in Section 5, based on morphological dilation. Then RCC relations are extended to bipolar fuzzy sets in Section 6. Finally, related works are discussed and compared with the proposed definitions in Section 7.

\section{Background on bipolar fuzzy sets and connectives}

In this section, we recall some useful definitions on bipolar fuzzy sets and basic connectives (negation, conjunction, disjunction, implication).

\subsection{Bipolar fuzzy sets}

As mentioned in the introduction, bipolar information has two components, one related to positive information, and one related to negative information. These pieces of information can take different forms, according to the application domain, such as preferences and constraints, observations and rules, possible and forbidden places for an object in space, etc. A simple example is displayed in Figure 1, where a positive information is that an object to be grasped by a robot is on the left of the red wall, while a negative information is that the robot is not allowed to go to the right of the red wall because this portion of space is filled with very fragile objects (in this example, positive information and negative information are of different nature, and may come from different sources). Note that the union of the two fuzzy domains does not cover the whole space, which leaves room for indetermination. By contrast, there is no overlap, ensuring consistency between positive and negative information.

Let us assume that bipolar information is represented by a pair $(\mu, v)$, where $\mu$ represents the positive information and $v$ the negative information, under a consistency constraint [24], which guarantees that the positive information is compatible with the constraints or rules expressed by the negative information. From a formal point of view, bipolar information can be represented in different settings. Here we consider the representation where $\mu$ and $v$ are membership functions to fuzzy sets, defined over a space $\mathcal{S}$ (for instance 


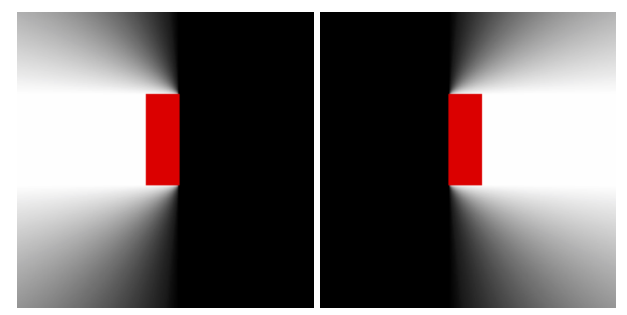

Figure 1: A spatial bipolar fuzzy set in a 2D space. Left: region to the left of the rectangle (positive information). Right: region to the right of the rectangle (negative information, corresponding to forbidden places). The membership degrees vary from 0 (black) to 1 (white).

the spatial domain, a set of potential options in preference modeling...), and the consistency constraint is expressed as $\forall x \in \mathcal{S}, \mu(x)+v(x) \leq 1$. The pair $(\mu, v)$ is then called a bipolar fuzzy set. As noticed e.g. in [21] and the subsequent discussion, bipolar fuzzy sets are formally equivalent (but with important differences in their semantics) to interval-valued fuzzy sets originally proposed in [38], where the membership of $x$ is expressed (using the same notations) as an interval $[\mu(x), 1-v(x)]$ of $[0,1]$ (hence implying the consistency constraint), and to intuitionistic fuzzy sets, where this consistency constraint was also proposed, along with the notion of membership and non-membership degrees [2]. These sets can also be interpreted as thick sets [16], where an ill-known set (or fuzzy set) is represented as an interval of sets (or fuzzy sets). All these are also special cases of L-fuzzy sets introduced in [26]. Despite these formal equivalences, since the semantics are very different [20, 21], we keep here the terminology of bipolarity.

Definition 1. A bipolar fuzzy set on $S$ is defined by an ordered pair of functions $(\mu, v)$ from $S$ into $[0,1]$ such that $\forall x \in \mathcal{S}, \mu(x)+v(x) \leq 1$ (consistency constraint).

We consider here that $\mu$ and $v$ are really two different functions, which may represent different types of information or may be issued from different sources (third type bipolarity according to [24]). However, this may also include the symmetric case, reducing the consistency constraint to a duality relation such as $v=1-\mu$. The proposed approach also differs from the one in [39] where bipolarity is encoded on $[-1,0] \times[0,1]$ for defining bipolar fuzzy logic.

For each point $x, \mu(x)$ defines the membership degree of $x$ (positive information) and $v(x)$ its nonmembership degree (negative information). This formalism allows representing both bipolarity and fuzziness. Since the positive information models what is possible, preferred, observed or experienced, and the negative information what is forbidden or impossible, the consistency constraint avoids contradictions between what is forbidden and what is possible (i.e. the potential solutions should be included in what is not forbidden or impossible). This is illustrated by the simple example in Figure 1. The set of bipolar fuzzy sets defined on $\mathcal{S}$ is denoted by $\mathcal{B}$.

Let us denote by $\mathcal{L}$ the set of ordered pairs of numbers $(a, b)$ in $[0,1]$ such that $a+b \leq 1$ (hence $(\mu, v) \in$ $\mathcal{B} \Leftrightarrow \forall x \in \mathcal{S},(\mu(x), v(x)) \in \mathcal{L})$. In all what follows, for each $(\mu, \nu) \in \mathcal{B}$, we will note $(\mu, v)(x)=(\mu(x), v(x))$ $(\in \mathcal{L}), \forall x \in \mathcal{S}$. Note that fuzzy sets can be considered as particular cases of bipolar fuzzy sets, either when $\forall x \in \mathcal{S}, v(x)=1-\mu(x)$, or when only one information is available, i.e. $(\mu(x), 0)$ or $(0,1-\mu(x))$. Furthermore, if $\mu$ (and $v$ ) only takes values 0 and 1 , then bipolar fuzzy sets reduce to classical sets.

Note that the interval $[0,1]$ is an arbitrary choice, and a more general setting could rely on L-fuzzy sets [26], by only assuming that $\mathcal{L}$ is a poset or a complete lattice. This line was followed in [37] for instance. Here we have chosen to keep $\mathcal{L}$ as presented above for simplifying the presentation and highlighting the bipolar nature of the information with its two components, but all what follows in this section and in the next one actually applies to more general forms of $\mathcal{L}$.

Let $\preceq$ be a partial ordering on $\mathcal{L}$ such that $(\mathcal{L}, \preceq)$ is a complete lattice. We denote by $\bigvee$ and $\bigwedge$ the supremum and infimum, respectively. The smallest element is denoted by $0_{\mathcal{L}}$ and the largest element by $1_{\mathcal{L}}$. We denote by $\succeq$ the reverse order, i.e. $\forall\left((a, b),\left(a^{\prime}, b^{\prime}\right)\right) \in \mathcal{L}^{2},(a, b) \succeq\left(a^{\prime}, b^{\prime}\right) \Leftrightarrow\left(a^{\prime}, b^{\prime}\right) \preceq(a, b)$. The partial 
ordering on $\mathcal{L}$ induces a partial ordering on $\mathcal{B}$, also denoted by $\preceq$ for the sake of simplicity:

$$
\left(\mu_{1}, v_{1}\right) \preceq\left(\mu_{2}, v_{2}\right) \text { iff } \forall x \in \mathcal{S},\left(\mu_{1}, v_{1}\right)(x) \preceq\left(\mu_{2}, v_{2}\right)(x) .
$$

Then $(\mathcal{B}, \preceq)$ is a complete lattice, for which the supremum and infimum are also denoted by $\bigvee$ and $\bigwedge$. For any family $\left\{\left(\mu_{i}, v_{i}\right), i \in I\right\}$ of elements of $\mathcal{B}$, where $I$ is an index set, we note $\left(\bigwedge_{i \in I}\left(\mu_{i}, v_{i}\right)\right)(x)=\bigwedge_{i \in I}\left(\mu_{i}(x), v_{i}(x)\right)$ and $\left(\bigvee_{i \in I}\left(\mu_{i}, v_{i}\right)\right)(x)=\bigvee_{i \in I}\left(\mu_{i}(x), v_{i}(x)\right)$, for all $x$ in $\mathcal{S}$. The smallest element of $(\mathcal{B}, \preceq)$ is the bipolar fuzzy set $0_{\mathcal{B}}=\left(\mu_{0}, v_{0}\right)$ taking value $0_{\mathcal{L}}$ at each point, and the largest element is the bipolar fuzzy set $1_{\mathcal{B}}=\left(\mu_{\mathbb{I}}, v_{\mathbb{I}}\right)$ always equal to $1_{\mathcal{L}}$.

\subsection{Bipolar connectives}

Let us now recall definitions and properties of connectives, that will be useful in the following and that extend to the bipolar case the connectives classically used in fuzzy set theory (see also [9]). In all what follows, increasingness and decreasingness are intended according to the partial ordering $\preceq$. Similar definitions can also be found e.g. in [18] in the case of interval-valued fuzzy sets or intuitionistic fuzzy sets, for a specific partial ordering (Pareto ordering), or in [37] in the more general setting of L-fuzzy sets [26].

Definition 2. Extensions of classical connectives to the bipolar setting are defined as follows:

- A negation, or complementation, on $\mathcal{L}$ is a decreasing operator $N$ such that $N\left(0_{\mathcal{L}}\right)=1_{\mathcal{L}}$ and $N\left(1_{\mathcal{L}}\right)=0_{\mathcal{L}}$. In this paper, we restrict ourselves to involutive negations, such that $\forall a \in \mathcal{L}, N(N(a))=a$ (these are the most interesting ones for mathematical morphology).

- A conjunction is an operator $C$ from $\mathcal{L} \times \mathcal{L}$ into $\mathcal{L}$ such that $C\left(0_{\mathcal{L}}, 0_{\mathcal{L}}\right)=C\left(0_{\mathcal{L}}, 1_{\mathcal{L}}\right)=C\left(1_{\mathcal{L}}, 0_{\mathcal{L}}\right)=$ $0_{\mathcal{L}}, C\left(1_{\mathcal{L}}, 1_{\mathcal{L}}\right)=1_{\mathcal{L}}$, and that is increasing in both arguments (i.e. $\forall\left(a_{1}, a_{2}, a_{1}^{\prime}, a_{2}^{\prime}\right) \in \mathcal{L}^{4}, a_{1} \preceq$ $a_{1}^{\prime}$ and $\left.a_{2} \preceq a_{2}^{\prime} \Rightarrow C\left(a_{1}, a_{2}\right) \preceq C\left(a_{1}^{\prime}, a_{2}^{\prime}\right)\right)$.

- A t-norm is a commutative and associative bipolar conjunction such that $\forall a \in \mathcal{L}, C\left(a, 1_{\mathcal{L}}\right)=$ $C\left(1_{\mathcal{L}}, a\right)=a$ (i.e. the largest element of $\mathcal{L}$ is the unit element of $C$ ). If only the property on the unit element holds, then $C$ is called a semi-norm.

- A disjunction is an operator $D$ from $\mathcal{L} \times \mathcal{L}$ into $\mathcal{L}$ such that $D\left(1_{\mathcal{L}}, 1_{\mathcal{L}}\right)=D\left(0_{\mathcal{L}}, 1_{\mathcal{L}}\right)=D\left(1_{\mathcal{L}}, 0_{\mathcal{L}}\right)=1_{\mathcal{L}}$, $D\left(0_{\mathcal{L}}, 0_{\mathcal{L}}\right)=0_{\mathcal{L}}$, and that is increasing in both arguments.

- A t-conorm is a commutative and associative bipolar disjunction such that $\forall a \in \mathcal{L}, D\left(a, 0_{\mathcal{L}}\right)=$ $D\left(0_{\mathcal{L}}, a\right)=a$ (i.e. the smallest element of $\mathcal{L}$ is the unit element of $\left.D\right)$.

- An implication is an operator I from $\mathcal{L} \times \mathcal{L}$ into $\mathcal{L}$ such that $I\left(0_{\mathcal{L}}, 0_{\mathcal{L}}\right)=I\left(0_{\mathcal{L}}, 1_{\mathcal{L}}\right)=I\left(1_{\mathcal{L}}, 1_{\mathcal{L}}\right)=1_{\mathcal{L}}$, $I\left(1_{\mathcal{L}}, 0_{\mathcal{L}}\right)=0_{\mathcal{L}}$ and that is decreasing in the first argument and increasing in the second argument. It follows that $\forall(a, b) \in \mathcal{L}, I\left(0_{\mathcal{L}},(a, b)\right)=1_{\mathcal{L}}$ and $I\left((a, b), 1_{\mathcal{L}}\right)=1_{\mathcal{L}}$.

In the following, we will call these connectives bipolar to make their instantiation on bipolar information explicit. Similarly, elements of $\mathcal{L}$ should be considered as pairs, quantifying the positive and negative parts of information. The properties of these connectives are detailed for instance in $[10,18]$, as well as the links between them. Let us mention of few of them, useful in the sequel:

- given a t-norm $C$ and a negation $N$, a t-conorm can be defined as

$$
D\left(\left(a_{1}, b_{1}\right),\left(a_{2}, b_{2}\right)\right)=N\left(C\left(N\left(\left(a_{1}, b_{1}\right)\right), N\left(\left(a_{2}, b_{2}\right)\right)\right)\right) ;
$$

- an implication $I$ induces a negation $N$ defined as $N((a, b))=I\left((a, b), 0_{\mathcal{L}}\right)$;

- an implication can be derived from a negation $N$ and a disjunction $D$ as

$$
I_{N}\left(\left(a_{1}, b_{1}\right),\left(a_{2}, b_{2}\right)\right)=D\left(N\left(\left(a_{1}, b_{1}\right)\right),\left(a_{2}, b_{2}\right)\right) ;
$$

- an implication can also be defined by residuation from a conjunction $C$ such that $\forall(a, b) \in \mathcal{L} \backslash$ $0_{\mathcal{L}}, C\left(1_{\mathcal{L}},(a, b)\right) \neq 0_{\mathcal{L}}$ as:

$$
I_{R}\left(\left(a_{1}, b_{1}\right),\left(a_{2}, b_{2}\right)\right)=\bigvee\left\{\left(a_{3}, b_{3}\right) \in \mathcal{L} \mid C\left(\left(a_{1}, b_{1}\right),\left(a_{3}, b_{3}\right)\right) \preceq\left(a_{2}, b_{2}\right)\right\}
$$


and we have $C\left(\left(a_{1}, b_{1}\right),\left(a_{3}, b_{3}\right)\right) \preceq\left(a_{2}, b_{2}\right) \Leftrightarrow\left(a_{3}, b_{3}\right) \preceq I\left(\left(a_{1}, b_{1}\right),\left(a_{2}, b_{2}\right)\right)$, expressing the adjunction property;

- if $C$ is a conjunction that admits $1_{\mathcal{L}}$ as unit element, then $C\left((a, b),\left(a^{\prime}, b^{\prime}\right)\right) \preceq(a, b) \wedge\left(a^{\prime}, b^{\prime}\right)$;

- if $I$ is an implication that admits $1_{\mathcal{L}}$ as unit element on the left, then $\left(a^{\prime}, b^{\prime}\right) \preceq I\left((a, b),\left(a^{\prime}, b^{\prime}\right)\right)$;

- if $I$ is an implication that admits $0_{\mathcal{L}}$ as unit element on the right, then $(a, b) \preceq I\left((a, b),\left(a^{\prime}, b^{\prime}\right)\right)$;

- a residual implication $I$ defined from a bipolar t-norm satisfies

$$
\begin{gathered}
I\left((a, b),\left(a^{\prime}, b^{\prime}\right)\right)=1_{\mathcal{L}} \Leftrightarrow(a, b) \preceq\left(a^{\prime}, b^{\prime}\right), \\
I\left(1_{\mathcal{L}},(a, b)\right)=(a, b) ; \\
\left(a^{\prime}, b^{\prime}\right) \preceq I\left((a, b),\left(a^{\prime}, b^{\prime}\right)\right) ;
\end{gathered}
$$

- from the adjunction property, the following properties are also derived:

$$
\begin{gathered}
C\left((a, b), I\left((a, b),\left(a^{\prime}, b^{\prime}\right)\right)\right) \preceq\left(a^{\prime}, b^{\prime}\right) \text { and }\left(a^{\prime}, b^{\prime}\right) \preceq I\left((a, b), C\left((a, b),\left(a^{\prime}, b^{\prime}\right)\right)\right) ; \\
C\left(I\left(\left(a_{1}, b_{1}\right),\left(a_{2}, b_{2}\right)\right),\left(a_{3}, b_{3}\right)\right) \preceq I\left(\left(a_{1}, b_{1}\right), C\left(\left(a_{2}, b_{2}\right),\left(a_{3}, b_{3}\right)\right)\right) ; \\
I\left(\left(a_{1}, b_{1}\right), I\left(\left(a_{2}, b_{2}\right),\left(a_{3}, b_{3}\right)\right)\right)=I\left(C\left(\left(a_{1}, b_{1}\right),\left(a_{2}, b_{2}\right)\right),\left(a_{3}, b_{3}\right)\right) ; \\
C\left(I\left(\left(a_{1}, b_{1}\right),\left(a_{2}, b_{2}\right)\right), I\left(\left(a_{2}, b_{2}\right),\left(a_{3}, b_{3}\right)\right)\right) \preceq I\left(\left(a_{1}, b_{1}\right),\left(a_{3}, b_{3}\right)\right) ;
\end{gathered}
$$

the last property expressing the $C$-transitivity of $I$.

Note that all properties derived from the adjunction are classical properties in any residuated lattice.

In the following we will mostly consider conjunctions which are bipolar t-norms, and the associated residual implications.

While all definitions and properties above hold for any partial ordering inducing a lattice structure, concrete instantiations of the connectives require to choose a specific ordering. The marginal partial ordering on $\mathcal{L}$, or Pareto ordering (by reversing the scale of negative information) is defined as:

$$
\left(a_{1}, b_{1}\right) \preceq\left(a_{2}, b_{2}\right) \text { iff } a_{1} \leq a_{2} \text { and } b_{1} \geq b_{2} \text {, }
$$

where $\leq$ and $\geq$ denote the classical ordering on real numbers. This ordering, often used in economics and social choice, has also been used for bipolar information [25], and for intuitionistic fuzzy sets (or interval valued fuzzy sets) e.g. in [18]. For this partial ordering, $(\mathcal{L}, \preceq)$ is a complete lattice. The greatest element is $1_{\mathcal{L}}=(1,0)$ and the smallest element is $0_{\mathcal{L}}=(0,1)$. The supremum and infimum are respectively defined as:

$$
\begin{aligned}
& \bigvee\left(\left(a_{1}, b_{1}\right),\left(a_{2}, b_{2}\right)\right)=\left(\max \left(a_{1}, a_{2}\right), \min \left(b_{1}, b_{2}\right)\right), \\
& \bigwedge\left(\left(a_{1}, b_{1}\right),\left(a_{2}, b_{2}\right)\right)=\left(\min \left(a_{1}, a_{2}\right), \max \left(b_{1}, b_{2}\right)\right)
\end{aligned}
$$

These equations extend directly to the supremum and infimum of any family of elements of $\mathcal{L}$.

In this paper, we restrict our developments to this partial ordering, as an example. Other partial orderings are discussed in [10], where dilations and erosions based on any ordering are proposed. As mentioned above, the partial order $\preceq$ induces a partial order on the set $\mathcal{B}$ of bipolar fuzzy sets.

Let us now mention a few connectives. In Definition 2, the monotony properties have now to be intended according to the Pareto ordering.

An example of negation, which will be used in the following, is the standard negation, defined by $N((a, b))=(b, a)$.

Two types of t-norms and t-conorms are considered in [18] (actually in the intuitionistic case) and will be considered here as well in the bipolar case (more details on different classes of operators can be found in [17]): 
1. The first class consists of operators called t-representable bipolar t-norms and t-conorms, which can be expressed using usual t-norms $t$ and t-conorms $T$ (see e.g. [23] for fuzzy connectives) as

$$
C\left(\left(a_{1}, b_{1}\right),\left(a_{2}, b_{2}\right)\right)=\left(t\left(a_{1}, a_{2}\right), T\left(b_{1}, b_{2}\right)\right),
$$

and

$$
D\left(\left(a_{1}, b_{1}\right),\left(a_{2}, b_{2}\right)\right)=\left(T\left(a_{1}, a_{2}\right), t\left(b_{1}, b_{2}\right)\right) .
$$

A typical example is obtained for $t=\min$ and $T=\max$. In the following we will use dual operators $t$ and $T$, although other choices are possible, as discussed e.g. in [29] for adjunction properties. Distributivity properties of implications over t-norms are further investigated in [3].

2. The second class includes bipolar Lukasiewicz operators, which are not t-representable:

$$
\begin{aligned}
& C_{W}\left(\left(a_{1}, b_{1}\right),\left(a_{2}, b_{2}\right)\right)=\left(\max \left(0, a_{1}+a_{2}-1\right), \min \left(1, b_{1}+1-a_{2}, b_{2}+1-a_{1}\right)\right), \\
& D_{W}\left(\left(a_{1}, b_{1}\right),\left(a_{2}, b_{2}\right)\right)=\left(\min \left(1, a_{1}+1-b_{2}, a_{2}+1-b_{1}\right), \max \left(0, b_{1}+b_{2}-1\right)\right) .
\end{aligned}
$$

The associated implications built from $C_{W}$ (or $D_{W}$ ) and $N$ or by residuation are the same and equal to:

$$
I_{W}\left(\left(a_{1}, b_{1}\right),\left(a_{2}, b_{2}\right)\right)=\left(\min \left(1, b_{1}+1-b_{2}, a_{2}+1-a_{1}\right), \max \left(0, a_{1}+b_{2}-1\right)\right) .
$$

In these equations, the positive part of $C_{W}$ is the usual Lukasiewicz t-norm $t_{L}$ of $a_{1}$ and $a_{2}$ (i.e. the positive parts of the input bipolar values). The negative part of $D_{W}$ is the usual Lukasiewicz t-norm of the negative parts $\left(b_{1}\right.$ and $\left.b_{2}\right)$ of the input values. Hence these operators are called pessimistic t-norm and optimistic t-conorm, respectively, in [17] ( $C_{W}$ and $D_{W}$ are actually two examples of such operators). Denoting by $T_{L}$ the usual Lukasiewicz t-conorm, we observe that the negative part of $C_{W}$ is equal to $\min \left(T_{L}\left(b_{1}, 1-a_{2}\right), T_{L}\left(b_{2}, 1-a_{1}\right)\right)$. Moreover, $1-a_{2}$ is the fuzzy negation of the negative part of $N\left(a_{2}, b_{2}\right)$. A similar interpretation holds for the positive part of $D_{W}$. Moreover, taking as negation $N(a, b)=(b, a)$, the bipolar Lukasiewicz operators satisfy properties similar to the non-contradiction and excluded-middle, i.e. $C_{W}((a, b), N(a, b))=0_{\mathcal{L}}$ and $D_{W}((a, b), N(a, b))=1_{\mathcal{L}}$. We also have $I_{W}((a, b),(a, b))=1_{\mathcal{L}}$.

\section{Basic set theoretical relations on bipolar fuzzy sets}

While an operation between two bipolar fuzzy sets (their intersection for example) can be computed using bipolar connectives (a conjunction for example, applied to every element of the support of the bipolar fuzzy sets), providing a new bipolar fuzzy set, here we want to assess to which degree a relation is satisfied by two bipolar fuzzy sets (e.g. two which degree they intersect), which is a different question. In the classical setting, this degree would be 0 or 1 , and in the fuzzy setting, it would be a number in $[0,1]$. Here, inclusion and intersection are defined as bipolar numbers (i.e. in $\mathcal{L}$ ) (see e.g. [10] and the references therein), so as to keep track of the imprecise and bipolar nature of information.

Definition 3. A bipolar degree of inclusion of $\left(\mu_{1}, v_{1}\right)$ in $\left(\mu_{2}, v_{2}\right)$ is defined from a bipolar implication I as:

$$
\operatorname{Inc}\left(\left(\mu_{1}, v_{1}\right),\left(\mu_{2}, v_{2}\right)\right)=\bigwedge_{x \in \mathcal{S}} I\left(\left(\mu_{1}, v_{1}\right)(x),\left(\mu_{2}, v_{2}\right)(x)\right) .
$$

Definition 4. A bipolar degree of intersection of $\left(\mu_{1}, v_{1}\right)$ and $\left(\mu_{2}, v_{2}\right)$ is defined from a bipolar conjunction $C$ as:

$$
\operatorname{Int}\left(\left(\mu_{1}, v_{1}\right),\left(\mu_{2}, v_{2}\right)\right)=\bigvee_{x \in \mathcal{S}} C\left(\left(\mu_{1}, v_{1}\right)(x),\left(\mu_{2}, v_{2}\right)(x)\right)
$$

These definitions are direct extensions of the degrees of inclusion and intersection of fuzzy sets, defined as the infimum of a fuzzy implication and the supremum of a fuzzy conjunction, respectively. 
Proposition 1. The bipolar degrees of inclusion and intersection in Definitions 3 and 4 have the following properties:

- They are consistent with the corresponding definitions in the crisp and fuzzy cases.

- The bipolar degree of inclusion is an element of $\mathcal{L}$ and is decreasing in the first argument and increasing in the second one.

- The bipolar degree of intersection is an element of $\mathcal{L}$ and is increasing in both arguments. It is symmetrical if $C$ is commutative (in particular if $C$ is a bipolar t-norm).

- If the conjunction is t-representable (i.e. $C=(t, T)$ with $t$ a t-norm and $T$ the dual $t$-conorm), then we have: $\operatorname{Int}\left(\left(\mu_{1}, v_{1}\right),\left(\mu_{2}, v_{2}\right)\right)=\left(\mu_{\text {int }}\left(\mu_{1}, \mu_{2}\right), 1-\mu_{\text {int }}\left(1-v_{1}, 1-v_{2}\right)\right)$ where $\mu_{\text {int }}$ is the degree of intersection between fuzzy sets, defined as $\mu_{\text {int }}\left(\mu, \mu^{\prime}\right)=\sup _{x \in \mathcal{S}} t\left(\mu(x), \mu^{\prime}(x)\right)$.

Proof. The proof of these properties is direct from the properties of $I$ and $C$.

\section{Mathematical morphology on bipolar fuzzy sets}

Mathematical morphology on bipolar fuzzy sets was proposed for the first time in [5], by considering the complete lattice defined from the Pareto ordering. Then it was further developed, with additional properties, geometric aspects and applications to spatial reasoning, in $[6,8]$. The lexicographic ordering was considered too in [9]. In [10], any partial ordering was considered, and derived operators were also proposed. Similar work has been developed independently, in the setting of intuitionistic fuzzy sets and interval-valued fuzzy sets, also based on Pareto ordering (e.g. [30]). This group proposed an extension to L-fuzzy sets [37], besides its important contribution to connectives (e.g. [18]). Here, while relying on the general algebraic framework of mathematical morphology, where a dilation is an operator that commutes with the supremum and an erosion an operator that commutes with the infimum of the lattice $(\mathcal{B}, \preceq$ ) (inducing a number of important properties, see e.g. [12] and the liminal references therein), on the one hand, and on L-fuzzy sets [26] on the other hand, we restrict ourselves to the special case of bipolar fuzzy sets, according to Definition 1, and use the definitions proposed in $[5,10]$, in their particular form involving structuring elements. A structuring element is a binary bipolar relation $\left(\mu_{B}, v_{B}\right)$ between elements of $\mathcal{S}$ and its value $\left(\mu_{B}, v_{B}\right)(x, y)$, for $x \in \mathcal{S}, y \in \mathcal{S}$, represents the bipolar degree to which this relation is satisfied between $x$ and $y$. If $\mathcal{S}$ is endowed with a translation (for instance $\mathcal{S}$ is a subset of $\mathbb{R}^{n}$ or $\mathbb{Z}^{n}$, representing a spatial domain), then a typical example would be $\left(\mu_{B}, v_{B}\right)(x, y)=\left(\mu_{B}, v_{B}\right)(y-x)$, and the value of a structuring element at $y-x$ represents the value at point $y$ of the translation of the structuring element at point $x$.

Definition 5. Let $\left(\mu_{B}, v_{B}\right)$ be a bipolar fuzzy structuring element (in $\left.\mathcal{B}\right)$. The erosion of any $(\mu, v)$ in $\mathcal{B}$ by $\left(\mu_{B}, v_{B}\right)$ is defined from a bipolar implication I as:

$$
\forall x \in \mathcal{S}, \varepsilon_{\left(\mu_{B}, v_{B}\right)}((\mu, v))(x)=\bigwedge_{y \in \mathcal{S}} I\left(\left(\mu_{B}, v_{B}\right)(x, y),(\mu, v)(y)\right) .
$$

Definition 6. Let $\left(\mu_{B}, v_{B}\right)$ be a bipolar fuzzy structuring element (in $\left.\mathcal{B}\right)$. The dilation of any $(\mu, v)$ in $\mathcal{B}$ by $\left(\mu_{B}, v_{B}\right)$ is defined from a bipolar conjunction $C$ as:

$$
\forall x \in \mathcal{S}, \delta_{\left(\mu_{B}, v_{B}\right)}((\mu, v))(x)=\bigvee_{y \in \mathcal{S}} C\left(\left(\mu_{B}, v_{B}\right)(y, x),(\mu, v)(y)\right)
$$

These definitions are proved to provide bipolar fuzzy sets, and express erosion (respectively dilation), as a degree of inclusion (respectively intersection) of the translation (if defined on $\mathcal{S}$ ) of the structuring element and the bipolar fuzzy set to be transformed, according to Definitions 3 and 4. An example of dilation for a particular choice of $C$ will be given in the next section.

The properties of these definitions are detailed in [10]. In particular, we will exploit the following properties: 
- dilation commutes with the supremum of the lattice, and is increasing;

- dilation is extensive if and only if the origin of $\mathcal{S}$ completely belongs to the structuring element (i.e. with bipolar degree $(1,0))$, which means that the relation $\left(\mu_{B}, v_{B}\right)$ is reflexive. We will restrict ourselves to extensive dilations in the following, i.e. such that $(\mu, v) \preceq \delta(\mu, v)$;

- the two operations $\varepsilon$ and $\delta$ form an adjunction if and only if $I$ is the residuated implication of $C$ (i.e. $I$ and $C$ are adjoint);

- finally, these definitions are equivalent to the fuzzy definitions if no bipolarity is taken into account (the dilation of a fuzzy set $\mu$ by a structuring element $\mu_{B}$ is defined as $\delta_{\mu_{B}}(\mu)(x)=$ $\sup _{y \in \mathcal{S}} t\left(\mu_{B}(y, x), \mu(y)\right)$ where $t$ is a t-norm [7], and a similar expression for erosion).

\section{Adjacency between bipolar fuzzy sets based on mathematical morphology}

In this section, we extend our previous work on adjacency between fuzzy sets [13] to the case of bipolar fuzzy sets. The underlying idea is similar, and relies on the fact that two entities (e.g. objects in space) are adjacent if they do not intersect, but as soon as one of them is dilated, they do intersect. This is illustrated in the crisp non bipolar case in Figure 2.



Figure 2: Adjacency between two crisp non bipolar sets: the two sets in plain lines do not intersect; when an elementary dilation (dashed line) is applied to one of the sets, then they do intersect.

Definition 7. Let $\delta$ be a bipolar fuzzy dilation, $C$ a bipolar conjunction, $N$ a bipolar negation, and Int a bipolar degree of intersection (Definition 4). The adjacency between two bipolar fuzzy sets $\left(\mu_{1}, v_{1}\right)$ and $\left(\mu_{2}, v_{2}\right)$ is defined as a bipolar number in $\mathcal{L}$ as:

$$
\operatorname{Adj}\left(\left(\mu_{1}, v_{1}\right),\left(\mu_{2}, v_{2}\right)\right)=C\left(N\left(\operatorname{Int}\left(\left(\mu_{1}, v_{1}\right),\left(\mu_{2}, v_{2}\right)\right)\right), \operatorname{Int}\left(\left(\mu_{1}, v_{1}\right), \delta\left(\mu_{2}, v_{2}\right)\right)\right) .
$$

This definition formalizes the conjunction $(C()$.$) between the non-intersection of the two entities (N(\operatorname{Int}())$. and the intersection of one entity and the dilation of the other one (see Definition 2 for $C$ and $N$, and Definition 4 for the degree of intersection Int).

The dilation can be defined according to the application. For instance for applications in the spatial domain, we may define a structuring element representing the smallest discernable spatial unit, or the imprecision related to object or point positions. The dilation is then computed using this structuring element. The simplest one in a discrete domain would be composed of one central point and its neighbors according to a pre-defined discrete connectivity. It would then be a classical set.

Let us detail the case where $C$ is a t-representable conjunction [18], i.e. $C\left(\left(a_{1}, b_{1}\right),\left(a_{2}, b_{2}\right)\right)=\left(t\left(a_{1}, a_{2}\right), T\left(b_{1}, b_{2}\right)\right)$ where $t$ is a t-norm and $T$ is a t-conorm. We consider here the Pareto ordering and $N(a, b)=(b, a)$, and we denote by $\delta^{+}$and $\delta^{-}$the positive and negative parts of the dilation, respectively. For instance for the dilation of a bipolar fuzzy set $\left(\mu_{2}, v_{2}\right)$ by a structuring element $\left(\mu_{B}, v_{B}\right)$ we have:

$$
\begin{aligned}
\delta^{+}(x) & =\sup _{y \in \mathcal{S}} t\left(\mu_{B}(x, y), \mu_{2}(y)\right)=\delta_{\mu_{B}}\left(\mu_{2}\right)(x), \\
\delta^{-}(x) & =\inf _{y \in \mathcal{S}} T\left(v_{B}(x, y), v_{2}(y)\right)=\varepsilon_{1-v_{B}}\left(v_{2}\right)(x) .
\end{aligned}
$$


These equations show that the positive part of the dilation is the fuzzy dilation of $\mu$ (positive part of the bipolar fuzzy set) by $\mu_{B}$ (positive part of the structuring element), and its negative part is the fuzzy erosion of $v$ (negative part of the bipolar fuzzy set) by $1-v_{B}$ (negation of the negative part of the structuring element). An illustration of a simple bipolar fuzzy set and its dilation with a bipolar fuzzy structuring element is displayed in Figure 3.

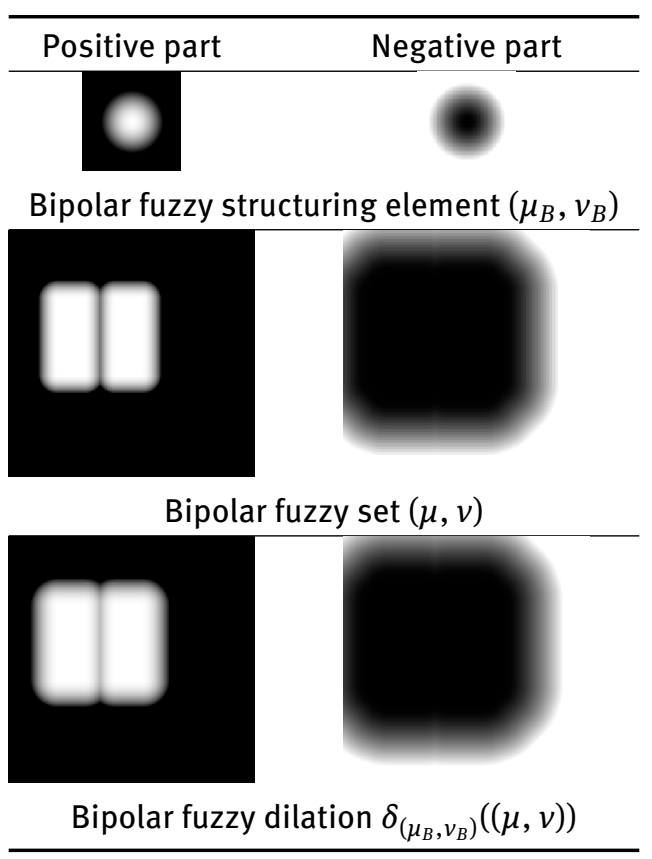

Figure 3: Illustration of a simple bipolar fuzzy dilation. High grey levels represent high membership values. Since the origin completely belongs to the structuring element (with bipolar degree equal to $1_{\mathcal{L}}$ ), the dilation is extensive (i.e. $(\mu, v) \preceq$ $\left.\delta_{\left(\mu_{B}, v_{B}\right)}((\mu, v))\right)$, which is illustrated by a larger positive part and a reduced negative part.

Proposition 2. For a t-representable conjunction defined from a t-norm $t$ and its dual t-conorm $T$, we have ( $\mu_{\text {int }}$ being the classical degree of intersection of fuzzy sets):

$$
\operatorname{Adj}\left(\left(\mu_{1}, v_{1}\right),\left(\mu_{2}, v_{2}\right)\right)=\left(t\left(1-\mu_{\text {int }}\left(1-v_{1}, 1-v_{2}\right), \mu_{\text {int }}\left(\mu_{1}, \delta^{+}\right)\right), T\left(\mu_{\text {int }}\left(\mu_{1}, \mu_{2}\right), 1-\mu_{\text {int }}\left(1-v_{1}, 1-\delta^{-}\right)\right)\right) .
$$

Proof. The proof results from a direct development of Equation 12, using the properties of Int (see Proposition 1), and the fact that $t$ and $T$ are dual, i.e. $\forall(a, b) \in[0,1]^{2}, T(a, b)=1-t(1-a, 1-b)$.

This result has an interpretation that corresponds to the intuition:

1. the positive part of $\operatorname{Adj}\left(\left(\mu_{1}, v_{1}\right),\left(\mu_{2}, v_{2}\right)\right)$ evaluates to which extent $1-v_{1}$ and $1-v_{2}$ do not intersect, and $\mu_{1}$ and $\delta^{+}$do intersect;

2. the negative part of $\operatorname{Adj}\left(\left(\mu_{1}, v_{1}\right),\left(\mu_{2}, v_{2}\right)\right)$ evaluates to which extent $\mu_{1}$ and $\mu_{2}$ intersect, or $1-v_{1}$ and $1-\delta^{-}$do not intersect;

where "and" is modeled by the t-norm $t$ and "or" by the t-conorm $T$.

The non-representable case, for instance for Lukasiewicz bipolar conjunction $C_{W}$ (see Equation 7), can be developed in a similar way. The interpretation is slightly more complicated and may be less directly intuitive.

Other properties are expressed in the following proposition.

Proposition 3. The adjacency defined in Definition 7 has the following properties: 
- it is symmetric if $C$ is commutative (e.g. $C$ is a bipolar t-norm) and the structuring element is symmetric (i.e. $\left.\forall(x, y) \in \mathcal{S}^{2},\left(\mu_{B}, v_{B}\right)(x, y)=\left(\mu_{B}, v_{B}\right)(y, x)\right)$;

- it is invariant under geometric transformations such as translation and rotation (if $\mathcal{S}$ is a spatial domain, e.g. a subset of $\mathbb{R}^{n}$ or $\mathbb{Z}^{n}$ );

- it is consistent with the definitions of adjacency in the binary case and in the fuzzy case.

Proof. The proof of the symmetry relies on the fact that a commutative conjunction commutes with the supremum, from which we derive that $\operatorname{Int}\left(\left(\mu_{1}, v_{1}\right), \delta\left(\mu_{2}, v_{2}\right)\right)=\operatorname{Int}\left(\delta\left(\mu_{1}, v_{1}\right),\left(\mu_{2}, v_{2}\right)\right)$. This explains why it is enough to dilate one of the bipolar fuzzy sets.

The proof of the other properties is straightforward.

Note that this relation is not considered in other works such as $[14,15,27,33]$. Due to the indetermination in the objects and in the relations, in some cases the adjacency relation may be close to relations such as "nearly overlap" in [14]. However, as mentioned in the introduction, the proposed model is more general since it is not restricted to objects with imprecise or broad boundaries (which are the ones considered in these other works), as illustrated in some of the examples below.

Example 1. A few typical adjacency situations are illustrated in Figure 4. For the sake of simplicity, degrees in the positive and negative parts are not represented, and only the bipolarity is explicitly displayed.

- In case (a) in this figure, the two positive parts are adjacent, and the indeterminate parts are overlapping, thus resulting in a strong indeterminacy in the spatial relation. The proposed definition provides a bipolar number where both positive and negative parts are less than 1, which reflects the ambiguity of this configuration.

- In case (b), the positive part of the adjacency is equal to 0 , showing that the relation is less satisfied than in case (a), as expected.

- The relation is best satisfied in case (c), with no ambiguity, which is reflected by the $(1,0)=1_{\mathcal{L}}$ bipolar value provided by the proposed definition.

- In case (d), the two negative parts of the sets are the same. This is an illustration where the bipolarity does not correspond to broad boundaries. Here the resulting bipolar adjacency value is $(0,1)=0_{\mathcal{L}}$, which corresponds to the fact that the possible region for the two objects is very large (the left halfplane), with potentially no adjacency between them because of overlapping.

- Finally, in case (e), the negative parts are different half-planes, and this situation is close to the perfect adjacency case. The largest value $(1,0)$ would be obtained if the two positive regions were strictly adjacent.

As a concrete example, the spatial entities in the two last situations may correspond to brain structures situated in the same hemisphere (then $v$ is the contra-lateral hemisphere), or in different hemispheres. Let us illustrate this, with the example of a slice of a magnetic resonance image (MRI) of the brain, in Figure 5. Assume we want to assess some topological relations among some of the structures delineated in this figure, under partial and imprecise information about them. For instance, we have a possible region for the left putamen, illustrated in Figure 6 (a), which is imprecise. The negative information is a quite large region in the right hemisphere (Figure 6 (b)). In a similar way, a possible region for the right Thalamus is shown in Figure $6(c)$, with the same negative information as for the left putamen (Figure $6(b)$ ). The computation of topological relations between these representations (and of other spatial relations) may then be used to guide the recognition of these structures in the image.

\section{RCC relations extended to bipolar fuzzy sets}

In this section, we propose extensions of the now classical RCC relations [32] to bipolar fuzzy sets. The connection predicate, a reflexive and symmetrical relation, is usually denoted by $C$, so we will denote conjunctions 


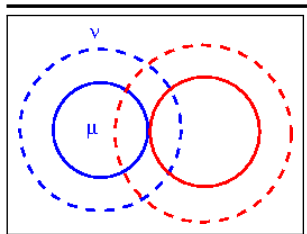

(a)

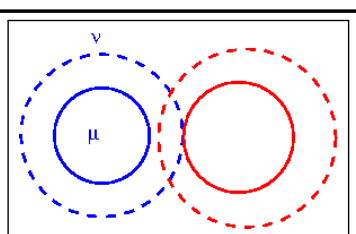

(b)

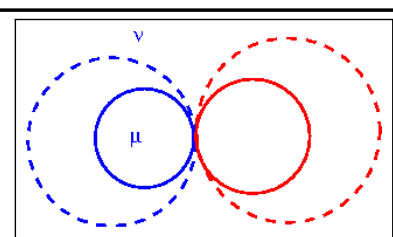

(c)

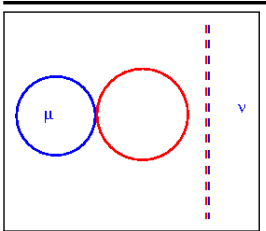

(d)

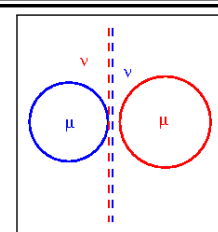

(e)

Figure 4: A few typical situations illustrating the adjacency between bipolar fuzzy sets in the spatial domain. The positive part $(\mu)$ is inside the plain lines, and the negative part $(v)$ is outside the dashed lines. For the sake of simplicity, membership degrees are not represented. The blue lines correspond to $\left(\mu_{1}, v_{1}\right)$ and the red ones to $\left(\mu_{2}, v_{2}\right)$.

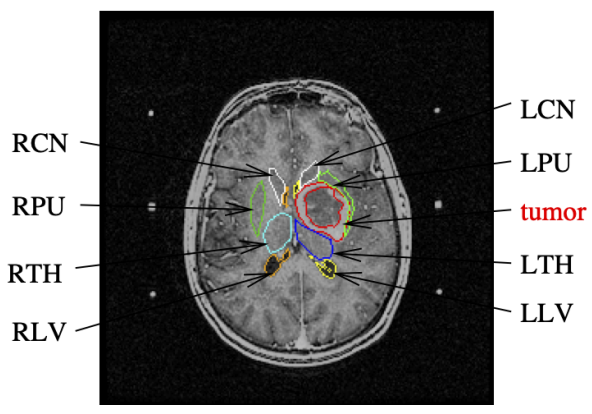

Figure 5: A slice of a 3D MRI brain image, with a few structures: left and right lateral ventricles (LLV and RLV), caudate nuclei (LCN and RCN), putamen (LPU and RPU) and thalamus (LTH and RTH). A ring-shaped tumor is present in the left hemisphere (the usual "left is right" convention is adopted for the visualization).

and bipolar t-norms by Conj in all this section. As detailed in [1], approaches for mereotopology differ depending on the interpretation of the connection and the properties of the considered regions (closed, open...).

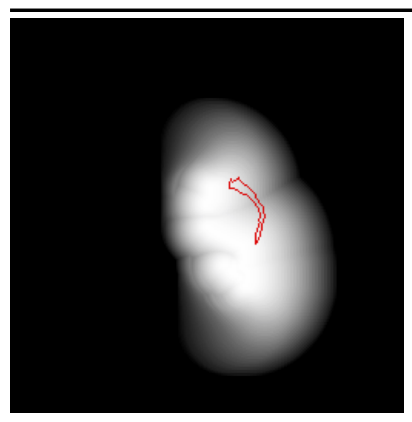

(a)

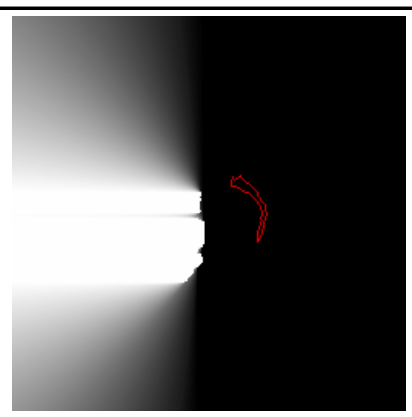

(b)

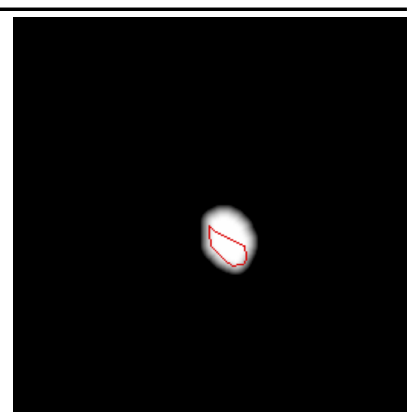

(c)

Figure 6: Bipolar fuzzy representations of two brain structures. (a) Positive information for the left putamen. (b) Negative information (for the two structures). (c) Positive information for the left thalamus. The contour of the two structures are superimposed on these fuzzy regions for visualization purpose, but are not part of the bipolar fuzzy representations. 


\subsection{Using connectives}

A first approach consists of a direct formal extension of the equations on classical sets, similar to the extension proposed in [34] for the fuzzy case. The proposed extension relies on the connectives and bipolar degrees of intersection and inclusion (see Sections 2 and 3). Thus we start with a bipolar connection predicate $C$, as defined abstractly below. To simplify notations, bipolar fuzzy sets are denoted by capital letters $(A, B \ldots \in \mathcal{B})$, but still represent pairs of positive and negative parts. Note that we do not refer to points here, and consider bipolar fuzzy sets as abstract entities. We will come back to concrete domains in Section 6.4 .

Definition 8. A bipolar connection predicate is defined as a mapping $C: \mathcal{B} \times \mathcal{B} \rightarrow \mathcal{L}$ such that $\forall(A, B) \in$ $\mathcal{B}^{2}, C(A, A)=1_{\mathcal{L}}, C(A, B)=C(B, A)$, i.e. $C$ is reflexive and symmetric.

Let us first recall the definitions of the RCC relations, expressed in first order logic for classical sets [32]. They are summarized in Table 1.

\begin{tabular}{|l|l|l|}
\hline$D C(x, y)$ & $x$ is disconnected from $y$ & $\neg C(x, y)$ \\
$P(x, y)$ & $x$ is a part of $y$ & $\forall z, C(z, x) \rightarrow C(z, y)$ \\
$P P(x, y)$ & $x$ is a proper part of $y$ & $P(x, y) \wedge \neg P(y, x)$ \\
$E Q(x, y)$ & $x$ is identical with $y$ & $P(x, y) \wedge P(y, x)$ \\
$O(x, y)$ & $x$ overlaps $y$ & $\exists z, P(z, x) \wedge P(z, y)$ \\
$D R(x, y)$ & $x$ is discrete from $y$ & $\neg O(x, y)$ \\
$P O(x, y)$ & $x$ partially overlaps $y$ & $O(x, y) \wedge \neg P(x, y) \wedge \neg P(y, x)$ \\
$E C(x, y)$ & $x$ is externally connected to & $C(x, y) \wedge \neg O(x, y)$ \\
& $y$ & \\
$T P P(x, y)$ & $x$ is a tangential proper part & $P P(x, y) \wedge \exists z[E C(z, x) \wedge E C(z, y)$ \\
& of $y$ & \\
$N T P P(x, y)$ & $x$ is a non tangential proper & $P P(x, y) \wedge \neg \exists z[E C(z, x) \wedge E C(z, y)]$ \\
& part of $y$ & \\
\hline
\end{tabular}

Table 1: RCC relations defined from a connection predicate $C$ (reflexive and symmetric relation) [32]. Spatial entities are denoted by $x, y, z$, the symbols $\rightarrow, \neg$, $\wedge$ denote the usual connectives of first order logic, and $\exists$ and $\forall$ the usual existential and universal quantifiers.

In the proposed definition below, the main idea is to replace logical symbols in Table 1 by their bipolar extensions: $\wedge$ becomes a conjunction Conj, $\neg$ a bipolar negation $N, \rightarrow$ a bipolar implication $I$. The existential and universal quantifiers are translated by $\bigvee$ and $\Lambda$, respectively. Now, when spatial entities become bipolar fuzzy sets, then the spatial relations are no more usual binary relations or predicates, but they hold up to some degree, which is also bipolar, hence an element of $\mathcal{L}$ (as for $C$ in Definition 8).

Definition 9. Let I be a bipolar implication, Conj a bipolar t-norm and $N$ a bipolar negation, according to Definition 2. The RCC relations on bipolar fuzzy sets are defined from a bipolar connection $C$ (Definition 8) as bipolar degrees of satisfaction (i.e. mappings from $\mathcal{B} \times \mathcal{B}$ into $\mathcal{L}$ ) as follows:

- Part: $P(A, B)=\bigwedge_{Z \in \mathcal{B}} I(C(A, Z), C(B, Z))$;

- Overlaps: $O(A, B)=\bigvee_{Z \in \mathcal{B}} \operatorname{Conj}(P(Z, A), P(Z, B))$;

- Non tangential part: $N T P(A, B)=\bigwedge_{Z \in \mathcal{B}} I(C(Z, A), O(Z, B))$;

- Disconnected: $D C(A, B)=N(C(A, B))$;

- Proper part: $P P(A, B)=\bigwedge(P(A, B), N(P(B, A))$ ) (or any bipolar conjunction Conj instead of $\bigwedge$ in this definition and the next ones);

- Equals: $E Q(A, B)=\bigwedge(P(A, B), P(B, A))$; 
- Distinct regions: $D R(A, B)=N(O(A, B))$;

- Partially overlaps: $P O(A, B)=\bigwedge(O(A, B), N(P(B, A)), N(P(A, B)))$;

- Externally connected: $E C(A, B)=\bigwedge(C(A, B), N(O(A, B)))$;

- Tangential proper part: $T P P(A, B)=\bigwedge(P P(A, B), N(N T P(A, B)))$;

- Non tangential proper part: $N T P P(A, B)=\bigwedge(P P(A, B), N T P(A, B))$.

\subsection{Using morphological dilations}

Another approach relies on the parthood predicate $P$ as starting point. Then we can directly derive the five relations $O, P P, E Q, D R, P O$ as above, thus leading to the five basic relations of RCC-5, i.e. $D R, P O, E Q, P P$ and $P P^{-1}$, where ${ }^{-1}$ denotes the inverse relation (i.e. $P P^{-1}(A, B)=P P(B, A)$ ). To get more relations, considering also whether objects touch each other, as in RCC-8 (where the eight basic relations are $D C, E C, T P P, T P P^{-1}$, $P O, E Q, N T P P, N T P P^{-1}$ ), in Definition 9 the predicate $C$ is involved. Here we propose another set of definitions, based on an extensive dilation $\delta$ (defined abstractly here, without referring to points of the underlying space). These links between RCC relations and mathematical morphology have been suggested in [12] in the crisp case (on classical sets), and further developed in the crisp discrete case in [28]. In the sequel, a dilation will be an operator $\delta: \mathcal{B} \rightarrow \mathcal{B}$ which commutes with the supremum (i.e. the classical abstract definition as in any lattice). We will need the dilation be extensive, i.e. $\forall A \in \mathcal{B}, A \preceq \delta(A)$.

Definition 10. A bipolar parthood predicate is defined as a mapping $P: \mathcal{B} \times \mathcal{B} \rightarrow \mathcal{L}$ such that $\forall(A, B) \in$ $\mathcal{B}^{2}, P(A, A)=1_{\mathcal{L}}$, i.e. $P$ is reflexive, and $\forall(A, B, C) \in \mathcal{B}^{3}$, $\operatorname{Conj}(P(A, B), P(B, C)) \preceq P(A, C)$, i.e. $P$ is Conjtransitive.

The properties imposed to $P$ in this definition are natural, since $P$ defined from $C$ (Section 6.1) will be shown to have these properties (Section 6.3).

Definition 11. Given a parthood predicate $P$ (supposed to be reflexive) and an extensive dilation $\delta$, the relations $O, P P, E Q, D R, P O$ are defined as in Definition 9, and the other ones, again mappings from $\mathcal{B} \times \mathcal{B}$ into $\mathcal{L}$, are defined as follows:

- $D C(A, B)=\bigwedge(D R(A, B), D R(A, \delta(B)))=\bigwedge(D R(A, B), D R(\delta(A), B)) ;$

- $E C(A, B)=\bigwedge(D R(A, B), O(A, \delta(B)))=\bigwedge(D R(A, B), O(\delta(A), B))$;

- TPP(A,B) $=\bigwedge(P P(A, B), O(\delta(A), N(B)))$, or, alternatively, $T P P(A, B)=\bigwedge(P P(A, B), N(P(\delta(A), B)))$;

- NTPP(A,B) $=\bigwedge(P(A, B), P(\delta(A), B))$.

These definitions are simple to implement (in concrete domains, see Section 6.4) and provide very concise expressions.

\subsection{Properties}

For crisp and fuzzy sets, the proposed definitions reduce to the existing ones in the crisp [32] and fuzzy cases [34], which is a desired consistency property. Moreover, the following properties hold, which are similar to the ones in the crisp and fuzzy cases.

Proposition 4. The relations in Definitions 9 and 11, for a residual implication I and the associated t-norm Conj, have the following properties:

1. the relations $O, D C, E Q, D R, P O, E C$ are symmetric;

2. the relations $P$ (in Definition 9), $O, E Q$ are reflexive;

3. the relations $P P, D C, D R, P O, E C, T P P$ and $N T P P$ are irreflexive;

4. $P$ in Definition 9 is Conj-transitive; 
5. if $P(A, B)=P(B, A)=1_{\mathcal{L}}$, then $E Q(A, B)=1_{\mathcal{L}}$, and for Definition $9 C(A, B)=C(B, A)=1_{\mathcal{L}}$;

6. $T P P \preceq P P\left(\right.$ i.e. $\left.\forall(A, B) \in \mathcal{B}^{2}, T P P(A, B) \preceq T P(A, B)\right), N T P P \preceq P P, P P \preceq P, E Q \preceq P, P O \preceq O, P \preceq O$, $E C \preceq D R, D C \preceq D R$, and for Definition $9 O \preceq C$ and $E C \preceq C$, leading to the lattice illustrated in Figure 7;

7. $\forall(A, B) \in \mathcal{B}^{2}, D_{W}(D C(A, B), C(A, B))=D_{W}(D R(A, B), C(A, B))=1_{\mathcal{L}}$ (for Definition 9), and $D_{W}(D R(A, B), O(A, B))=$ $1_{\mathcal{L}} ;$

8. if $C$ is increasing in both arguments, then $P$ is decreasing in the first argument and increasing in the second one (for Definition 9), and if $P$ is supposed to have these monotony properties in Definition 11, then $O$ is increasing, $D C$ and $D R$ are decreasing, NTPP and PP are decreasing in the first argument and increasing in the second one, and NTPP reduces to $N T P P(A, B)=P(\delta(A), B)$.

Proof.

1. The symmetry of $O, D C, E Q, D R, P O, E C$ is obvious from the definitions of these relations.

2. $\forall A \in \mathcal{B}, P(A, A)=\bigwedge_{Z \in \mathcal{B}} I(C(A, Z), C(A, Z))=1_{\mathcal{L}}\left(\right.$ since $\left(I(a, b),\left(a^{\prime}, b^{\prime}\right)\right)=1_{\mathcal{L}}$ iff $\left.(a, b) \preceq\left(a^{\prime}, b^{\prime}\right)\right)$, hence $P$ is reflexive. The reflexivity of $O$ and $E Q$ follows.

3. $\forall A \in \mathcal{B}, P P(A, A)=\bigwedge(P(A, A), N(P(A, A)))=\bigwedge\left(1_{\mathcal{L}}, 0_{\mathcal{L}}\right)=0_{\mathcal{L}}$, hence $P P$ is irreflexive. $\forall A \in \mathcal{B}, D C(A, A)=$ $N(C(A, A))=0_{\mathcal{L}}$ since $C$ is reflexive, hence $D C$ is irreflexive. Similarly $D R$ is irreflexive since $O$ is reflexive. The irreflexivity of $P O$ follows from the reflexivity of $P$ and $O$, the irreflexivity of $E C$ follows from the reflexivity of $C$ and $O$, and the irreflexivity of $T P P$ and $N T P P$ follows from the irreflexivity of $P P$.

4. The Conj-transitivity of $P$ follows from the one of $I$ (see Section 2.2):

$$
\begin{aligned}
\operatorname{Conj}\left(P\left(A_{1}, A_{2}\right), P\left(A_{2}, A_{3}\right)\right) & =\operatorname{Conj}\left(\bigwedge_{Z \in \mathcal{B}} I\left(C\left(A_{1}, Z\right), C\left(A_{2}, Z\right)\right), \bigwedge_{Z \in \mathcal{B}} I\left(C\left(A_{2}, Z\right), C\left(A_{3}, Z\right)\right)\right) \\
& \preceq \bigwedge_{Z \in \mathcal{B}} \operatorname{Conj}\left(I\left(C\left(A_{1}, Z\right), C\left(A_{2}, Z\right)\right), I\left(C\left(A_{2}, Z\right), C\left(A_{3}, Z\right)\right)\right) \\
& \preceq \bigwedge_{Z \in \mathcal{B}} I\left(C\left(A_{1}, Z\right), C\left(A_{3}, Z\right)\right) \\
& \preceq P\left(A_{1}, A_{3}\right)
\end{aligned}
$$

5. Obvious from the definitions.

6. $T P P \preceq P P, N T P P \preceq P P, P P \preceq P, E Q \preceq P, P O \preceq O, E C \preceq C$ follow directly from the definitions. $E C(A, B)=\bigwedge(C(A, B), N(O(A, B)))=\bigwedge(C(A, B), D R(A, B))$ hence $E C \preceq D R$.

Since $P$ is reflexive, we have $P(A, B) \preceq \operatorname{Conj}(P(A, A), P(A, B)) \preceq \bigvee_{Z} \operatorname{Conj}(P(Z, A), P(Z, B)) \preceq O(A, B)$. From the reflexivity and symmetry of $C$, the fact that $I\left(1_{\mathcal{L}}, Z\right)=Z$, and the fact that $\operatorname{Conj}(X, I(X, Y)) \preceq Y$, we have:

$$
\begin{aligned}
O(A, B) & =\bigvee_{Z} \operatorname{Conj}(P(Z, A), P(Z, B)) \\
& =\bigvee_{Z} \operatorname{Conj}\left(\bigwedge_{Z^{\prime}} I\left(C\left(Z, Z^{\prime}\right), C\left(A, Z^{\prime}\right)\right), \bigwedge_{Z^{\prime}} I\left(C\left(Z, Z^{\prime}\right), C\left(B, Z^{\prime}\right)\right)\right) \\
& \preceq \bigvee_{Z} \operatorname{Conj}(I(C(Z, Z), C(A, Z)), I(C(Z, A), C(B, A))) \\
& \preceq \bigvee_{Z} \operatorname{Conj}(C(A, Z), I(C(A, Z), C(A, B))) \\
& \preceq C(A, B)
\end{aligned}
$$

hence $O \preceq C$

$D C \preceq D R$ follows from $O \preceq C$ and the fact that $N$ reverses the order.

7. The result is derived directly from the definitions and from the expression of the Lukasiewicz disjunction (Equation 8). Indeed, by denoting $C(A, B)=\left(C^{+}, C^{-}\right)$, and since $D C(A, B)=N(C(A, B)$ ), we have $D_{W}(D C(A, B), C(A, B))=D_{W}\left(\left(C^{-}, C^{+}\right),\left(C^{+}, C^{-}\right)\right)=1_{\mathcal{L}}$ (excluded middle). The second equality comes from the monotony of $D_{W}$ and from $D C \preceq D R$. Finally, $D_{W}(D R(A, B), O(A, B))=D_{W}(N(O(A, B)), O(A, B))=1_{\mathcal{L}}$ (excluded middle). 
8. Monotony properties follow from the monotony properties of $\operatorname{Conj}, I, \wedge, \bigvee$ and $\delta$.

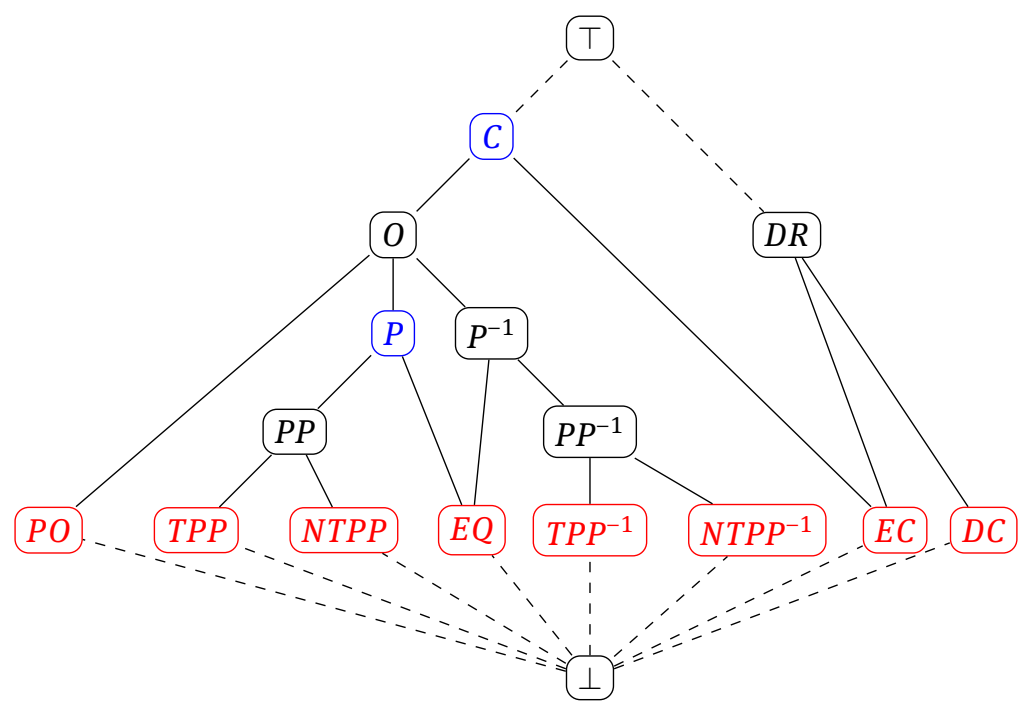

Figure 7: Lattice of bipolar RCC relations (similar to the classical one). The leaves (in red) are the eight basic relations of RCC-8 (among which $D R, P O, P P, E Q$ and $P P^{-1}$ are the five basic relations of RCC-5). They are obtained from $C$ in the connectivebased approach, and from $P$ and an extensive dilation in the morphological approach.

\subsection{Interpretations in concrete domains}

In Definitions 9 and 11, the relations are abstract (i.e. concepts in the language of ontologies) and do not make any reference to elements of $\mathcal{S}$ (points). Reasoning can then be performed on any bipolar (spatial) entities. Now, if we move to the concrete domain $\mathcal{S}$, which is often necessary for practical applications, then we can assign to each abstract bipolar fuzzy set an interpretation in $\mathcal{S}$. Interpretations of the RCC relations then require concrete definitions of $C$, or $P$ and $\delta$. This can be achieved in different ways:

- $C$ can be defined as a degree of intersection (Definition 4);

- $C$ can be defined as a closeness relation (as in [35] for the fuzzy case), for instance from a dilation and a degree of intersection;

- $P$ can be defined as an inclusion relation (Definition 3) and $\delta$ as a dilation with a given structuring element containing the origin of $\mathcal{S}$, i.e. defined as a reflexive relation (Definition 6). Note that the morphological definition of $E C$ is then exactly the proposed definition for adjacency in Section 5.

Proposition 5. Depending on the chosen interpretation, the following properties hold:

1. If $C$ is defined as a degree of intersection, i.e. $\forall(A, B) \in \mathcal{B} \times \mathcal{B}, C(A, B)=\bigvee_{x \in \mathcal{S}} \operatorname{Conj}(A(x), B(x))$ where Conj is a bipolar t-norm, then

- $P$ is a degree of inclusion, i.e. $\forall(A, B) \in \mathcal{B} \times \mathcal{B}, P(A, B)=\bigwedge_{x \in \mathcal{S}} I(A(x), B(x))$, where $I$ is the residual implication of Conj, and

$-O=C$. 
2. If $C$ is defined as a closeness relation $R$, e.g. $C(A, B)=\bigvee_{x \in \mathcal{S}} \operatorname{Conj}\left(A(x), \delta_{R}(B)(x)\right)$, where $\delta_{R}$ is a dilation expressing the closeness relation $R\left(\delta_{R}(B)(x)\right.$ then expresses the bipolar degree to which $x$ is close to $\left.B\right)$, then

- $P(A, B)$ is the degree of inclusion of $\varepsilon_{R} \delta_{R}(A)$ in $\varepsilon_{R} \delta_{R}(B)$, where $\varepsilon_{R}$ is the adjoint erosion of $\delta_{R}$, and

- $O(A, B)$ is the degree of intersection of $\varepsilon_{R} \delta_{R}(A)$ and $\varepsilon_{R} \delta_{R}(B)$.

3. If $P$ is defined as a degree of inclusion, $O=C$ as a degree of intersection, and I from $N$ and a disjunction $D$, then the two variants of TPP in Definition 11 are equivalent.

Proof.

1. From the properties of implication and conjunction (see Section 2.2), it is easy to show that for any $Z$, we have

$$
I(C(A, Z), C(B, Z)) \succeq \bigwedge_{x \in \mathcal{S}} I(A(x), B(x)) .
$$

Hence $P(A, B) \succeq \bigwedge_{x \in \mathcal{S}} I(A(x), B(x))$.

Conversely, by limiting $Z$ to the bipolar fuzzy sets reduced to one point, i.e. $Z_{x}$ such that $Z_{x}\left(x^{\prime}\right)=1_{\mathcal{L}}$ if $x^{\prime}=x$ and $0_{\mathcal{L}}$ otherwise, we have

$$
\begin{aligned}
P(A, B) & \preceq \bigwedge_{x \in \mathcal{S}} I\left(\bigvee_{x^{\prime} \in \mathcal{S}} \operatorname{Conj}\left(A\left(x^{\prime}\right), Z_{x}\left(x^{\prime}\right)\right), \bigvee_{x^{\prime} \in \mathcal{S}} \operatorname{Conj}\left(B\left(x^{\prime}\right), Z_{x}\left(x^{\prime}\right)\right)\right) \\
& \preceq \bigwedge_{x \in \mathcal{S}} I(A(x), B(x))
\end{aligned}
$$

Finally, $P(A, B)=\bigwedge_{x \in \mathcal{S}} I(A(x), B(x))$, which is the degree of inclusion of $A$ in $B$.

Note that this proof is quite similar to the one proposed in [35] for the fuzzy case.

From Proposition 4, $O \preceq C$ always hold. Now, using the same set of $Z_{x}$ as above, we have:

$$
\begin{aligned}
O(A, B) & \succeq \bigvee_{x \in \mathcal{S}} \operatorname{Conj}\left(\bigwedge_{x^{\prime} \in \mathcal{S}} I\left(Z_{x}\left(x^{\prime}\right), A\left(x^{\prime}\right)\right), \bigwedge_{x^{\prime} \in \mathcal{S}} I\left(Z_{x}\left(x^{\prime}\right), B\left(x^{\prime}\right)\right)\right) \\
& \succeq \bigvee_{x \in \mathcal{S}} \operatorname{Conj}(A(x), B(x)) \\
& \succeq C(A, B)
\end{aligned}
$$

and finally $O=C$.

2. The proof in the fuzzy case of [35] extends directly to the bipolar case.

3. We have $O(\delta(A), N(B))=\bigvee_{x \in \mathcal{S}} \operatorname{Conj}(\delta(A)(x), N(B)(x))$, and

$$
\begin{aligned}
N(P(\delta(A), B)) & =N\left(\bigwedge_{x \in \mathcal{S}} I(\delta(A)(x), B(x))\right) \\
& =\bigvee_{x \in \mathcal{S}} N(D(N(\delta(A))(x), B(x))) \\
& =\bigvee_{x \in \mathcal{S}} \operatorname{Conj}(\delta(A)(x), N(B)(x)) .
\end{aligned}
$$

Example 2. Let us assume that $P$ is defined concretely as an inclusion degree, as in Definition 3, using an implication I defined from a representable disjunction $D$ built from $(t, T)$ where $t$ is a $t$-norm and $T$ its dual t-conorm. Then for all $\left(a_{1}, b_{1}\right),\left(a_{2}, b_{2}\right)$ in $\mathcal{L}$, we have:

$$
I\left(\left(a_{1}, b_{1}\right),\left(a_{2}, b_{2}\right)\right)=D\left(N\left(a_{1}, b_{1}\right),\left(a_{2}, b_{2}\right)\right)=D\left(\left(b_{1}, a_{1}\right),\left(a_{2}, b_{2}\right)\right)=\left(T\left(b_{1}, a_{2}\right), t\left(a_{1}, b_{2}\right)\right)
$$

Let us set $A=\left(\mu_{1}, v_{1}\right), B=\left(\mu_{2}, v_{2}\right)$. Then

$$
P(A, B)=\bigwedge_{x \in \mathcal{S}}\left(T\left(v_{1}(x), \mu_{2}(x)\right), t\left(\mu_{1}(x), v_{2}(x)\right)\right)
$$


Example 3. In the configuration of Figure 8, where again only the bipolarity of $\left(\mu_{1}, v_{1}\right)$ and $\left(\mu_{2}, v_{2}\right)$ is displayed, Definition 11 with $P$ defined as a degree of inclusion leads to $P P\left(\left(\mu_{1}, v_{1}\right),\left(\mu_{2}, v_{2}\right)\right)=(1,0)=1_{\mathcal{L}}$ and $\operatorname{TPP}\left(\left(\mu_{1}, v_{1}\right),\left(\mu_{2}, v_{2}\right)\right)=(1,0)=1_{\mathcal{L}}$.

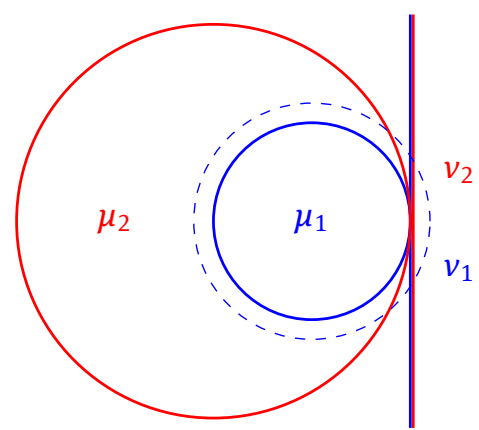

Figure 8: Interpretation in the spatial domain of two bipolar sets $\left(\mu_{1}, v_{1}\right)$ (blue) and $\left(\mu_{2}, v_{2}\right)$ (red), for which $P P\left(\left(\mu_{1}, v_{1}\right),\left(\mu_{2}, v_{2}\right)\right)=\operatorname{TPP}\left(\left(\mu_{1}, v_{1}\right),\left(\mu_{2}, v_{2}\right)\right)=(1,0)=1_{\mathcal{L}}$. The dashed blue circle represents the positive part of the dilation of $\left(\mu_{1}, v_{1}\right)$.

\section{Related work and comparison}

The work developed in this paper deals with the third type of bipolarity, as defined in [24], where both positive information and negative information are important, not necessarily directly linked together (besides the consistency constraint), and possibly issued from different sources of information. Obviously the proposed definitions also apply to the other types of bipolarity.

While the definitions can remain abstract and conceptual, their interpretations in a concrete domain can have various semantics. For spatial reasoning, the concrete domain is the space (denoted by $\mathcal{S}$ in the previous sections). A bipolar fuzzy set can then represent information on where an object can be and where it cannot be, on where an object could be searched and where it should not, epistemic knowledge on some object (e.g. we know that there is a tumor in the left hemisphere of the brain, and that it cannot be in the right hemisphere), a region where the attention should be focused on, a region satisfying some constraints and preferences (for example to position an object, to guide the displacement of a robot, to focus a radiotherapy on a tumor while avoiding organs at risk, etc.). In all these examples, the positive and negative parts of the bipolar fuzzy sets play asymmetric roles. The definitions introduced in this paper provide a toolbox to assess relations between such regions, to explore the space, to solve constraint satisfactions problems, etc.

This context is more general than existing work. Classical RCC relations [32] apply to spatial entities, which are arbitrary and abstract, but neither fuzzy nor bipolar. Fuzzy spatial relations $[4,35]$ apply to fuzzy sets defined in the spatial domain, without the notion of bipolarity. The egg-yolk formalism [15] aims at handling indeterminate boundary, and objects are defined as two concentric regions (yolk surrounded by egg white). This can be extended to the fuzzy case by considering two membership functions, one included in the other one. By contrast, the bipolar fuzzy sets do not have this interpretation as boundary (see for instance the example in Figure 8). The notion of boundary vagueness (as in the egg-yolk model) is distinguished from the locational vagueness in [27]. A semantic close to the one of egg-yolk is the one of crisping introduced in [33]. A lower crisping is the area definitely in the region, while an upper crisping is the area possibly in the region, with an inclusion constraint of the lower crisping in the upper crisping. This idea is close to the notion of rough sets [31] and to that of thick sets [16, 20]. Connection is then handled with three truth values, in the Lukasiewicz algebra: two regions are definitely connected if each crisping of one region is connected with each crisping of the other region, they are possibly connected if one crisping of one region is connected to one crisping of the other region, and they are not connected if none of the crisping of one region is connected 
to any crisping of the other region. Broad boundaries are also considered in [14], and again this model is more restricting than bipolar fuzzy sets. Spatial reasoning with this model is based on all possible situations of intersection between the interior of a region, its closure and its broad boundary (all being crisp regions). Again the model cannot handle situations such as in Figure 8 which have no interpretation in terms of boundary. Although this model is similar to the egg-yolk model, the intended semantics are different. In the egg-yolk model, a region is a crisper version of another one, hence representing ignorance, while the broad boundaries represent a transitional area around the region (this transition being supposed to be narrow), representing vagueness.

In all these approaches, mostly the imprecision on the regions or objects is considered (in particular with different models of their boundaries), hence dealing only with the positive part of the information on these regions. The potential negative part is never considered as another source of information. Hence the proposed approach substantially extends the type of spatial information that can be handled. Moreover, the proposed formulation is generic, whatever the spatial configuration of the considered bipolar fuzzy sets, and does not require to enumerate all possible situations, in contrast to these previous works.

In Section 6.4 we mentioned a few potential interpretations of basic spatial relations, whatever the spatial domain for interpreting the objects. A specific interpretation of regions in the 2D discrete case was proposed in [28], for classical sets (neither fuzzy nor bipolar). Nevertheless it is interesting for its links with mathematical morphology, and the derived efficient algorithms for computing RCC-5 and RCC-8 relations, and for the fact that specificities of the discrete case lead to the definition of slightly different relations. For instance there is a clear distinction between $E C$ (adjacent with no intersection), $D C$ (at least one pixel separation between the two regions), and neighborhood connection (one pixel separation between two regions), these relations having simple expressions based on dilations. A similar approach could be developed based on the definitions proposed in this paper, instantiated in a discrete space $\mathcal{S}$.

\section{Conclusion}

In this paper, we proposed original definitions of algebraic relations (intersection, inclusion, adjacency and RCC relations) between bipolar fuzzy sets, using mathematical morphology operators, in particular dilation. This problem had never been addressed before, and could be useful in spatial reasoning, but also in other domains, such as preference modeling, where preferences can be considered as bipolar fuzzy sets. The proposed relations can be instantiated with various interpretations, in particular in concrete domains defined as the spatial domain, whatever its dimension. Although we relied on bipolar semantics in this paper, the proposed definitions and properties apply more generally to any type of functions, taking values in a poset or in a complete lattice, including the case of L-fuzzy sets, and pave the way for other potential interpretations and applications. It would be useful to further develop the semantics attached to the notion of spatial bipolarity, for instance taking inspiration from the enlightening discussions in [20, 21], and develop their counterpart when it comes to spatial information and its specificities.

Future work aims at exploring such applications, with concrete examples. This would require to define concretely the spatial bipolar fuzzy sets, which was not addressed in this paper. Positive and negative regions can be provided by some prior information, coming from different sources, or by some operations applied to regions of space. Inspiration could be found in signal and image processing using maxitive kernels or interval-valued kernels, such as in [19], where filtering by such kernels provides a representation of a signal which could be interpreted as an interval-valued fuzzy set. Extensions to higher dimension and to asymmetric bipolarity could be investigated. Other aspects will be investigated as well, such as different partial ordering relations. The proposed definitions apply for any ordering, but the particular choice of one ordering (Pareto, lexigographic, etc.) is important from a semantic point of view (see e.g. the discussion in [10] for bipolar fuzzy mathematical morphology). Another interesting direction concerns composition tables and reasoning, extending the work in the crisp and fuzzy cases [32, 36]. The Conj-transitivity of $P$ in Proposition 4 is a first 
step towards establishing such composition tables. Finally, other types of spatial relations can be addressed, such as directional relations, as well as other relations specific to the 3D case.

\section{Acknowledgments:}

This work has been partly supported by the French ANR LOGIMA project, and the French-Brazilian ANRFAPESP STAP project.

\section{References}

[1] Aiello, M., Pratt-Hartmann, I., van Benthem (Ed.), J.: Handbook of Spatial Logic. Springer (2007).

[2] Atanassov, K. T.: Intuitionistic Fuzzy Sets. Fuzzy Sets and Systems, 20, 87-96 (1986).

[3] Baczynski, M.: On the Distributivity of Implication Operations over t-Representable t-Norms Generated from Strict t-Norms in Interval-Valued Fuzzy Sets Theory. In: Information Processing and Management of Uncertainty in Knowledge-Based Systems (IPMU 2010), pp. 637-646 (2010).

[4] Bloch, I.: Fuzzy spatial relationships for image processing and interpretation: a review. Image and Vision Computing 23(2), 89-110 (2005).

[5] Bloch, I.: Dilation and Erosion of Spatial Bipolar Fuzzy Sets. In: International Workshop on Fuzzy Logic and Applications WILF 2007. vol. LNAI 4578, pp. 385-393. Genova, Italy (2007).

[6] Bloch, I.: Bipolar Fuzzy Mathematical Morphology for Spatial Reasoning. In: Wilkinson, M., Roerdink, J. (eds.) International Symposium on Mathematical Morphology ISMM'09. vol. LNCS 5720, pp. 24-34. Groningen, The Netherlands (2009).

[7] Bloch, I.: Duality vs. Adjunction for Fuzzy Mathematical Morphology and General Form of Fuzzy Erosions and Dilations. Fuzzy Sets and Systems 160, 1858-1867 (2009).

[8] Bloch, I.: Bipolar Fuzzy Spatial Information: Geometry, Morphology, Spatial Reasoning. In: Jeansoulin, R., Papini, O., Prade, H., Schockaert, S. (eds.) Methods for Handling Imperfect Spatial Information, pp. 75-102. Springer (2010).

[9] Bloch, I.: Lattices of fuzzy sets and bipolar fuzzy sets, and mathematical morphology. Information Sciences 181, 2002-2015 (2011).

[10] Bloch, I.: Mathematical morphology on bipolar fuzzy sets: general algebraic framework. International Journal of Approximate Reasoning 53, 1031-1061 (2012).

[11] Bloch, I.: Topological relations between bipolar fuzzy sets based on mathematical morphology. In: International Symposium on Mathematical Morphology (ISMM 2017). vol. LNCS 10225, pp. 40-41. Fontainebleau, France (2017).

[12] Bloch, I., Heijmans, H., Ronse, C.: Mathematical Morphology. In: Aiello, M., Pratt-Hartman, I., van Benthem, J. (eds.) Handbook of Spatial Logics, chap. 13, pp. 857-947. Springer (2007).

[13] Bloch, I., Maître, H., Anvari, M.: Fuzzy Adjacency between Image Objects. International Journal of Uncertainty, Fuzziness and Knowledge-Based Systems 5(6), 615-653 (1997).

[14] Clementini, E., Felice, O.D.: Approximate Topological Relations. International Journal of Approximate Reasoning 16, 173-204 (1997).

[15] Cohn, A.G., Gotts, N.M.: The egg-yolk representation of regions with indeterminate boundaries. In: Burough, P., Frank, A. (eds.) Geographical Objects with Indeterminate Boundaries, GISDATA 2, 171-187 (1996).

[16] Desrochers, B., Jaulin, L.: Thick set inversion. Artificial Intelligence, 249, 1-18 (2017).

[17] Deschrijver,G., Cornelis, C.: Representability in interval-valued fuzzy set theory. International Journal of Uncertainty Fuzziness and Knowledge Based Systems, 15(3), 345-361 (2007).

[18] Deschrijver, G., Cornelis, C., Kerre, E.: On the Representation of Intuitionistic Fuzzy t-Norms and t-Conorms. IEEE Transactions on Fuzzy Systems 12(1), 45-61 (2004).

[19] Destercke, S., Rico, A., Strauss, O.: Approximating General Kernels by Extended Fuzzy Measures: Application to Filtering. In: International Conference on Information Processing and Management of Uncertainty in Knowledge-Based Systems (IPMU), vol. CCIS 1238, pp. 112-123 (2020).

[20] Dubois, D., Jaulin, L., Prade, H.: Thick Sets, Multiple-Valued Mappings, and Possibility Theory. In: Statistical and Fuzzy Approaches to Data Processing, with Applications to Econometrics and Other Areas, Studies in Computational Intelligence, vol. 892, Springer, pp. 101-109 (2020).

[21] Dubois, D., Gottwald, S., Hajek, P., Kacprzyk, J., Prade, H.: Terminology Difficulties in Fuzzy Set Theory - The Case of “Intuitionistic Fuzzy Sets". Fuzzy Sets and Systems 156, 485-491 (2005).

[22] Dubois, D., Prade, H. (Eds.): Special issue on bipolar representations of information and preference. International Journal of Intelligent Systems 23(8-10) (2008).

[23] Dubois, D., Prade, H.: Fuzzy Sets and Systems: Theory and Applications, Academic Press, New York (1980). 
[24] Dubois, D., Prade, H.: An Overview of the Asymmetric Bipolar Representation of Positive and Negative Information in Possibility Theory. Fuzzy Sets and Systems 160, 1355-1366 (2009).

[25] Fargier, H., Wilson, N.: Algebraic structures for bipolar constraint-based reasoning. In: ECSQARU. vol. LNCS 4724, pp. 623634 (2007).

[26] Goguen, J.: L-fuzzy sets. Journal of Mathematical Analysis and Applications 18(1), 145-174 (1967).

[27] Hazarika, S., Cohn, A.: A taxonomy for spatial vagueness: An alternative egg-yolk interpretation. In: Spatial Vagueness, Uncertainty and Granularity Symposium. Ogunquit, Maine, USA (2001).

[28] Landini, G., Galton A., Randell, D., and Fouad., S.: Novel applications of discrete mereotopology to mathematical morphology. Signal Processing: Image Communications 76, 109-117 (2019).

[29] Medina, J.: Adjoint Pairs on Interval-Valued Fuzzy Sets. In: Information Processing and Management of Uncertainty in Knowledge-Based Systems (IPMU 2010), pp. 430-439 (2010).

[30] Mélange, T., Nachtegael, M., Sussner, P., Kerre, E.: Basic Properties of the Interval-Valued Fuzzy Morphological Operators. In: IEEE World Congress on Computational Intelligence WCCI 2010. pp. 822-829. Barcelona, Spain (2010).

[31] Pawlak. Z.: Rough Sets. International Journal of Information and Computer Science, 11(5), 341-356 (1982).

[32] Randell, D., Cui, Z., Cohn, A.: A Spatial Logic based on Regions and Connection. In: Nebel, B., Rich, C., Swartout, W. (eds.) Principles of Knowledge Representation and Reasoning KR’92. pp. 165-176. Kaufmann, San Mateo, CA (1992).

[33] Roy, A.J., Stell, J.G.: Spatial relations between indeterminate regions. International Journal of Approximate Reasoning 27(3), 205-234 (2001)

[34] Schockaert, S., De Cock, M., Cornelis, C., Kerre, E.E.: Fuzzy region connection calculus: Representing vague topological information. International Journal of Approximate Reasoning 48(1), 314-331 (2008).

[35] Schockaert, S., De Cock, M., Cornelis, C., Kerre, E.E.: Fuzzy region connection calculus: An interpretation based on closeness. International Journal of Approximate Reasoning 48(1), 332-347 (2008).

[36] Schockaert, S., De Cock, M., Kerre, E.E.: Spatial reasoning in a fuzzy region connection calculus. Artificial Intelligence 173(2), 258-298 (2009).

[37] Sussner, P., Nachtegael, M., Mélange, T., Deschrijver, G., Esmi, E., Kerre, E.: Interval-Valued and Intuitionistic Fuzzy Mathematical Morphologies as Special Cases of L-Fuzzy Mathematical Morphology. Journal of Mathematical Imaging and Vision 43(1), 50-71 (2012).

[38] Zadeh, L. A.: The Concept of a Linguistic Variable and its Application to Approximate Reasoning. Information Sciences 8 , 199-249 (1975).

[39] Zhang, W.R., Zhang, L.: YinYang bipolar logic and bipolar fuzzy logic. Information Sciences 165(3-4), 265-287 (2004). 